\title{
Diffusion and viscosity in a supercooled polydisperse system
}

\author{
Rajesh K. Murarka and Biman Bagchi* \\ Solid State and Structural Chemistry Unit, Indian Institute of Science, Bangalore, India 560012
}

\begin{abstract}
We have carried out extensive molecular dynamics simulations of a supercooled polydisperse Lennard-Jones liquid with large variations in temperature at a fixed pressure. The particles in the system are considered to be polydisperse in both size and mass. The temperature dependence of dynamical properties such as the viscosity $(\eta)$ and the self-diffusion coefficients $\left(D_{i}\right)$ of different size particles is studied. Both viscosity and diffusion coefficients show super-Arrhenius temperature dependence and fit well to the well-known Vogel-FulcherTammann equation. Within the temperature range investigated, the value of Angell's fragility parameter $(D$ $\approx 1.4$ ) classifies the present system as a very fragile liquid. The critical temperature for diffusion $\left(T_{o}^{D_{i}}\right)$ increases with the size of the particles. The critical temperature for viscosity $\left(T_{o}^{\eta}\right)$ is larger than that for diffusion, and sizable deviations appear for the smaller size particles, implying a decoupling of translational diffusion from viscosity in deeply supercooled liquids. Indeed, the diffusion shows markedly non-Stokesian behavior at low temperatures where a highly nonlinear dependence on size is observed. An inspection of the trajectories of the particles shows that at low temperatures the motions of both the smallest and largest size particles are discontinuous (jump type). However, the crossover from continuous Brownian to large length hopping motion takes place at shorter time scales for the smaller size particles.
\end{abstract}

\section{INTRODUCTION}

The rapid cooling of a liquid below its freezing temperature transforms it into a long-lived metastable amorphous solid or glass [1]. Understanding the dynamics of the system near the glass transition has been an intense field of research in the last few decades. There have been many experimental [2-7] as well as simulation studies [8-14] which focus on the dynamics of dense supercooled liquids well above the glass transition and also near the glass transition temperature $\left(T_{g}\right)$. The basic aim of all these studies was to characterize quantitatively the observed very complex dynamics of the system as it approaches the glass transition from above. Close to the glass transition, the shear viscosity $(\eta)$ and the microscopic structural relaxation time $(\tau)$ of the so-called fragile glass-forming liquids show divergence with a strongly non-Arrhenius temperature dependence [1]. This divergence is often well represented by the Vogel-FulcherTammann (VFT) equation:

$$
\eta(T)=A_{\eta} \exp \left[E_{\eta} /\left(T-T_{o}^{\eta}\right)\right]
$$

where $A_{\eta}$ and $E_{\eta}$ are temperature-independent constants and $T_{o}^{\eta}\left(<T_{g}\right)$ is the temperature at which $\eta$ diverges. Note that at low temperatures the increasingly slow dynamics of the so-called fragile liquids is simultaneously manifested in the stretched exponential decay of the stress correlation function (with a strongly temperature-dependent stretching parameter) $[15,16]$. The VFT dependence [Eq. (1)] is thus accompanied by strong nonexponential relaxation observed near the glass transition temperature.

\footnotetext{
*Author to whom correspondence should be addressed. Electronic address: bbagchi@sscu.iisc.ernet.in
}

The dramatic slowdown of the dynamics near the glass transition is not well understood and still remains the most challenging problem in the physics of glasses. Several theories have been proposed to understand the anomalous relaxation dynamics of deeply supercooled liquids. Although the ideal version of nonlinear mode coupling theory (MCT) [17] gives a microscopic picture of this slowing down, it predicts a structural arrest, i.e., a transition from ergodic to nonergodic behavior, at a critical temperature $T_{c}$, well above the laboratory glass transition temperature $T_{g}$. Near $T_{c}$, the importance of the influence of the potential energy landscape on the relaxation processes has now been widely accepted $[18,19]$, and strongly correlated jump motion is observed to be the dominant mode for mass transport [13,20-22]; this is not included in ideal MCT.

Another important characteristic feature in the dynamics of deeply supercooled liquids is the decoupling between translational diffusion and the shear viscosity of the medium [23-25]. At high temperature, over a wide range of liquid states, the translational diffusion is inversely proportional to viscosity, in accordance with the Stokes-Einstein (SE) relation given by

$$
D_{T}=\frac{k_{B} T}{C \pi \eta R},
$$

where $R$ is the spherical radius of the diffusing particle and $C$ is a numerical constant that depends on the hydrodynamic boundary condition. However, several recent experimental [26-28] and simulation studies [15,29-33] on strongly supercooled "fragile" glass-forming liquids have shown significant deviations from the SE relation. As the temperature is lowered toward $T_{g}$, it is found that the translational diffusion is larger than the value predicted by the SE relation, and in some cases even two to three orders of magnitude larger [23]. The enhanced diffusion at low temperatures is some- 
times explained in terms of a power law behavior $D_{T} \propto \eta^{-\alpha}$ with $\alpha<1[28,30]$. Both the experiments and the simulation studies have suggested that the enhancement of the translational diffusion coefficient is due to the spatially heterogeneous dynamics in deeply supercooled liquids $[2,27,28,31,32]$.

Computer simulations have played a key role in augmenting our understanding of various aspects of the dynamics of supercooled liquids from a microscopic viewpoint. Unfortunately, simple one-component systems such as soft or hard spheres or Lennard-Jones systems crystallize rapidly on lowering the temperature below the melting point $\left(T_{m}\right)$, and, therefore, cannot be utilized as a model for studying the complex dynamical behavior near the glass transition temperature. A natural way to avoid crystallization is to use binary mixtures of atoms with different diameters. A large number of molecular dynamics (MD) simulations have recently been carried out in supercooled model binary mixtures near the glass transition as well as below the glass transition temperature [8-12,16,21,22,34-36].

However, one is often interested in the consequences of the disorder introduced by the dissimilarity of the particles. Synthetic colloids, by their very nature, frequently exhibit considerable size polydispersity [37,38]. Polydispersity is also common in industrially produced polymers which always contain macromolecules with a range of chain length. Colloidal particles are an excellent model of hard spheres, and perhaps the simplest possible experimental system of interacting particles to study the glass transition. Several experiments [37] and simulations [39,40] have shown that the crystal phase of colloidal systems can exist as a thermodynamically stable phase only for polydispersities (standard deviation of the size distribution divided by the mean) less than a "terminal" value, in the range of $0.05-0.15$.

Interestingly, recent experiments on colloidal supercooled fluids and colloidal glasses allowed one to obtain information on the microscopic details of the dynamics of the individual particles [3]. These experiments have shown the presence of dynamic heterogeneity in deeply supercooled colloidal systems. The motion of the relatively fast-moving particles is found to be highly correlated and they form connected clusters whose size increases as one approaches the glass transition. More recently, Sear [41] carried out a MD simulation of a dense polydisperse hard sphere fluid to study the effect of polydispersity on the slow dynamics. The simulation results also show the clustering of the fast-moving particles in agreement with the experiments, although the dynamics appears to be less heterogeneous. The heterogeneous nature of the dynamics has also been observed in a Monte Carlo simulation study of polydisperse hard spheres close to the glass transition [42].

It is worth mentioning that, in addition to the short-range hard-core interaction, addition of a nonadsorbing soluble polymer in the stable colloidal suspension gives rise to a weak, long-range attraction between the colloidal particles by means of the depletion interaction [38]. Recently, the consequences of this attractive interaction on the glass transition have been nicely explained in a combined experimental, theoretical, and simulation study by Pham et al. [43]. Interest- ingly, with increase in the strength of the short-range attractive interaction, two qualitatively different glassy states are found with a reentrant glass transition line.

The size distribution in real colloids generally leads to a distribution in mass of the particles. The importance of the mass polydispersity on the dynamics of a realistic system having size polydispersity has recently been analyzed in a molecular dynamics simulation study of a Lennard-Jones (LJ) polydisperse fluid near the triple point of the corresponding monodisperse LJ system [44]. Polydispersity is commonly found in many systems of industrial applications, and to mimic the interparticle interactions the Lennard-Jones potential generally serves as a good starting model. Thus, it will be of general interest to study the impact of polydispersity on the dynamics of a deeply supercooled polydisperse fluid, where particles interact via the LJ potential. More importantly, this will enable us to compare the properties of the system with model binary LJ mixtures whose dynamics near the glass transition has been studied extensively in simulations.

In this study, we have performed extensive molecular dynamics simulations of a system of polydisperse LJ spheres with a continuous range of diameters and mass. The temperature dependence of dynamic properties such as the viscosity $(\eta)$ and the self-diffusion coefficients $\left(D_{i}\right)$ for different size particles is studied by varying the temperature $(T)$ over a large range at a constant high pressure $(P)$. Both the viscosity and diffusion show super-Arrhenius temperature dependence, and the calculated value of the fragility parameter $(D)$ shows that the present system is more fragile than the wellknown Kob-Andersen binary mixture [8]. The critical temperature obtained from the VFT fit to the diffusion $\left(T_{o}^{D_{i}}\right)$ shows strong dependency on the radius $\left(R_{i}\right)$ of the particles. In addition, the critical temperature obtained from the VFT fit to the viscosity $\left(T_{o}^{\eta}\right)$ is much higher when compared to those of the diffusion coefficients, where the deviation is largest for the smaller size particles. This clearly reflects the deviation from the Stokesian diffusion in the proximity of the glass transition temperature. Most interestingly, at lower temperatures the diffusion shows a highly nonlinear size dependence when plotted against the inverse of the radius $\left(R_{i}\right)$ of the particles. The reason for the breakdown of the StokesEinstein relation can be analyzed from the trajectories of the particles. We find that, at low temperature, hopping processes are the primary mode of particle diffusion for both the smaller and bigger size particles.

The organization of the rest of the paper is as follows. In the next section, we describe in detail the system studied here and the details of the simulations. The simulation results are analyzed and discussed in Sec. III. Finally, we end with some concluding remarks in Sec. IV.

\section{SYSTEM AND SIMULATION DETAILS}

We have performed a series of equilibrium isothermalisobaric ensemble $(N-P-T)$ molecular dynamics simulations in three dimensions of a system of $N=256$ particles of mean radius $\bar{\sigma}$ and mass $\bar{m}$ with polydispersity in both size and 
mass. The interaction between any two particles is modeled by means of a shifted-force Lennard-Jones pair potential, where the standard LJ potential is given by [45]

$$
u_{i j}^{L J}=4 \epsilon_{i j}\left[\left(\frac{\sigma_{i j}}{r_{i j}}\right)^{12}-\left(\frac{\sigma_{i j}}{r_{i j}}\right)^{6}\right]
$$

where $i$ and $j$ denote two different particles and $\sigma_{i j}=\left(\sigma_{i}\right.$ $\left.+\sigma_{j}\right) / 2$ with $\sigma_{i}, \sigma_{j}$ the diameters of the particles $i$ and $j$, respectively. In the shifted-force potential both the potential and force are continuous at a cutoff radius $r_{c}$, and we choose a value of $r_{c}=2.5 \bar{\sigma}$.

The polydispersity in size is introduced by random sampling from the Gaussian distribution of particle diameters $\sigma$ [44]:

$$
P(\sigma)=\frac{1}{\delta \sqrt{2 \pi}} \exp \left[-\frac{1}{2}\left(\frac{\sigma-\bar{\sigma}}{\delta}\right)^{2}\right],
$$

where $\delta$ is the width of the distribution. The standard deviation $(\delta)$ of the distribution divided by its mean $\bar{\sigma}$ gives a dimensionless parameter, the polydispersity index $s=\delta / \bar{\sigma}$. The simulations are carried out here for a fixed value of the polydispersity index $s=0.1$. The masses of the particles are varied accordingly, and we assume that the mass of a particle $i$ is scaled by its diameter as $m_{i}=\bar{m}\left(\sigma_{i} / \bar{\sigma}\right)^{3}$. The LJ energy parameter $\epsilon_{i j}$ is assumed to be the same for all particle pairs and is denoted as $\epsilon$. All the quantities in this study are given in reduced units, that is, length in units of $\bar{\sigma}$, temperature $T$ in units of $\epsilon / k_{B}$, pressure $P$ in units of $\epsilon / \bar{\sigma}^{3}$, and time in units of $\tau=\sqrt{\bar{m} \bar{\sigma}^{2} / \epsilon}$. Note that if one assumes argon units then $\tau=2.2 \mathrm{ps}$.

All simulations in the $N-P-T$ ensemble are performed using the Nosé-Hoover-Andersen method [46], where the external temperature $(T)$ is varied over a large range from 1.3 to 0.67 keeping the external pressure $(P)$ fixed at 10.0. Throughout the course of the simulations, the barostat and system's degrees of freedom are coupled to an independent Nosé-Hoover chain (NHC) [47] of thermostats, each of length 5. The extended system equations of motion are integrated using the reversible integrator method [48]. The higher order multiple time step method has been employed in the NHC evolution operator, which leads to stable energy conservation for non-Hamiltonian dynamical systems [49]. The extended system time scale parameter used in the calculations is taken to be 0.93 for $T \geqslant 1.0$ and 1.16 for $T<1.0$ for both the barostat and thermostats.

A time step of 0.001 is employed for $T \geqslant 1.0$ and 0.002 for $T<1.0$. The number of equilibration and data collection steps is also varied accordingly depending upon the temperature of the system. For $T \geqslant 1.0$, the number of equilibration steps is varied from $5 \times 10^{5}$ to $10^{6}$ and the number of data collection steps is $10^{6}$, whereas for $T<1.0$, the number of equilibration steps is varied from $5.0 \times 10^{5}$ to $2.0 \times 10^{6}$ and the number of data collection steps from $10^{6}$ to $2.5 \times 10^{7}$. At each temperature, all the dynamic quantities are averaged over five independent runs. Diffusion coefficients $\left(D_{i}\right)$ for

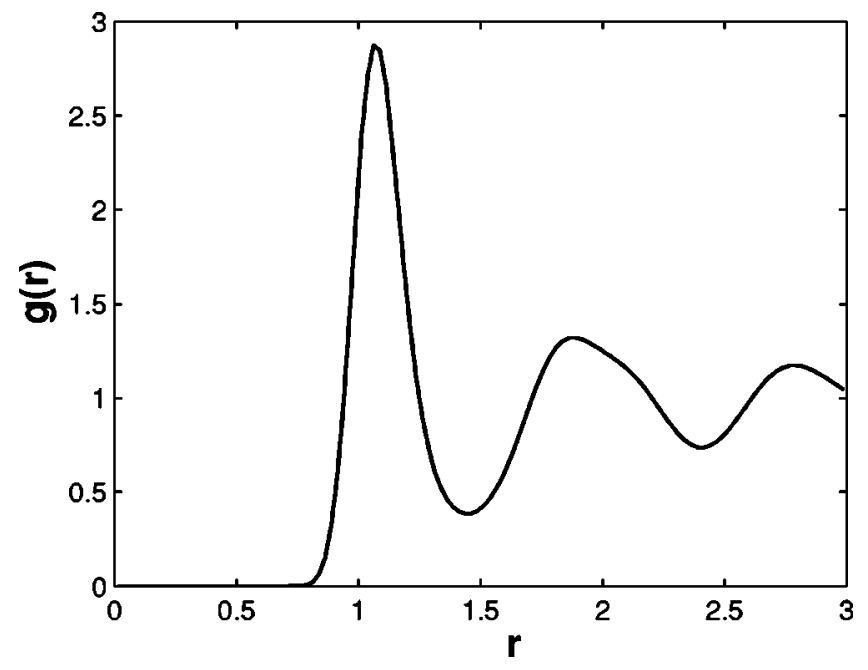

FIG. 1. The radial distribution function $g(r)$ of the system at $T=0.67$, the lowest temperature investigated.

the different size particles are calculated from the slope of the corresponding mean square displacements in the diffusive limit, and viscosity is calculated from the autocorrelation of the off-diagonal components of the microscopic stress tensor, via the standard Green-Kubo formula [50]. As the system is isotropic, we have taken an average over three different off-diagonal stress correlations for each of the five data sets.

\section{RESULTS AND DISCUSSION}

In order to make sure that there is no crystallization, we calculated the radial distribution functions $g(r)$ which describes the average structure of the fluid. The radial distribution function calculated for $T=0.67$, the lowest temperature investigated, is shown in Fig. 1. The decay of correlations with increase in distance is consistent with the absence of any long-range order, a characteristic feature of the fluid.

The plot of $\ln [\eta]$ as a function of inverse temperature $(1 / T)$ in Fig. 2(a) clearly shows a super-Arrhenius behavior of the viscosity. In Fig. 2(b) we show a VFT fit to the viscosity [Eq. (1)] by plotting $\ln [\eta]$ against $1 /\left(T-T_{o}^{\eta}\right)$, where $T_{o}^{\eta}$ is equal to 0.57 . As in other fragile liquids, it shows that the divergence of the viscosity is quite well described by the VFT equation. From the fitting we obtain the values of $A_{\eta}$ and $E_{\eta}$ as 2.0 and 0.81 , respectively. We also calculated the fragility parameter $\left(D=E_{\eta} / T_{o}^{\eta}\right)$ as defined by Angell [51]. Using the values of the fitting parameters $\left(E_{\eta}\right.$ and $\left.T_{o}^{\eta}\right)$ obtained within the temperature range investigated, its value is $\approx 1.42$. This classifies the present system as a very fragile liquid. Thus, the dense random packing of unequal size particles makes the present system more fragile when compared with a recent simulation study on a Kob-Andersen binary mixture $(D \approx 2.45)$ [16].

The temperature dependence of the average diffusion coefficient $\bar{D}$ of the system (averaged over all particles) is shown in Fig. 3(a) where $-\ln [\bar{D}]$ is plotted as a function of $1 / T$. Similar to what is observed for viscosity [Fig. 2(a)] the 

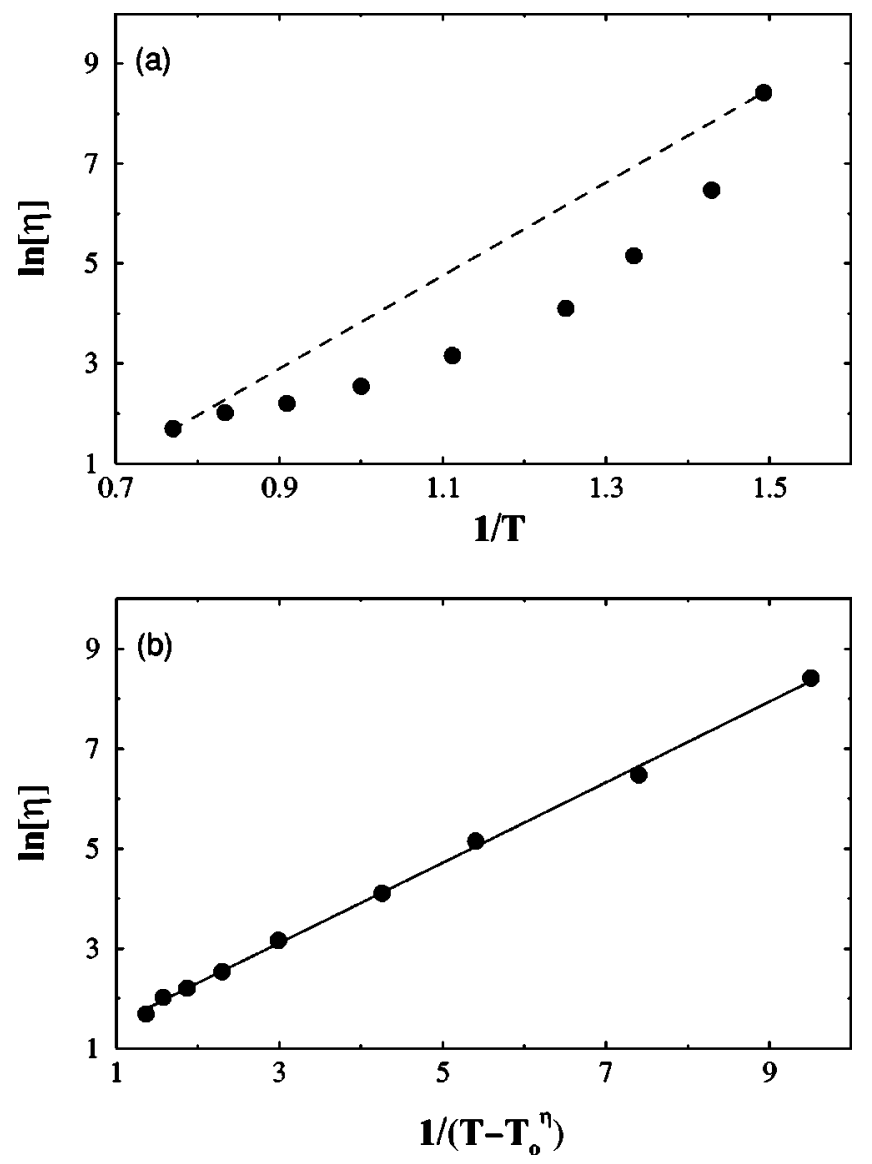

FIG. 2. Temperature dependence of the shear viscosity $(\eta)$. (a) $\ln [\eta]$ as a function of inverse temperature $(1 / T)$. The simulated values given by the solid circles show super-Arrhenius behavior. The dashed line gives a guideline to the Arrhenius behavior. (b) $\ln [\eta]$ against $1 /\left(T-T_{o}^{\eta}\right)$. The solid circles again represent the simulation results and the VFT fit is shown by the solid line. $T_{o}^{\eta}$ is found to be 0.57 . The slope $E_{\eta}$ and the intercept $\ln \left[A_{\eta}\right]$ obtained from the fit are 0.81 and 0.69 , respectively. Note that the viscosity $(\eta)$ is scaled by $\sqrt{\bar{m} \epsilon / \bar{\sigma}^{4}}$. For details, see the text.

diffusion coefficient $(\bar{D})$ shows a super-Arrhenius temperature dependence. In a polydisperse system, all the particles are unequal in size, so their diffusion coefficients also differ. We categorize the particles into different subsets where particles of diameters within $0.05 \bar{\sigma}$ are assumed to be members of the same subset. For the polydispersity index $s=0.1$, we find that the minimum and maximum diameters of the particles are $0.75 \bar{\sigma}$ and $1.25 \bar{\sigma}$, respectively. Thus subsets of particles with diameters in the ranges $0.75 \bar{\sigma}$ to $0.8 \bar{\sigma}$ and $1.2 \bar{\sigma}$ to $1.25 \bar{\sigma}$ correspond to the smallest and largest spheres, respectively. The diffusion coefficients for different subsets of particles are calculated at each temperature. It is well known that in deeply supercooled liquids the non-Arrhenius temperature dependence of the diffusion coefficient can be fitted by a VFT law:

$$
D_{i}(T)=A_{D_{i}} \exp \left[-E_{D_{i}} /\left(T-T_{o}^{D_{i}}\right)\right]
$$
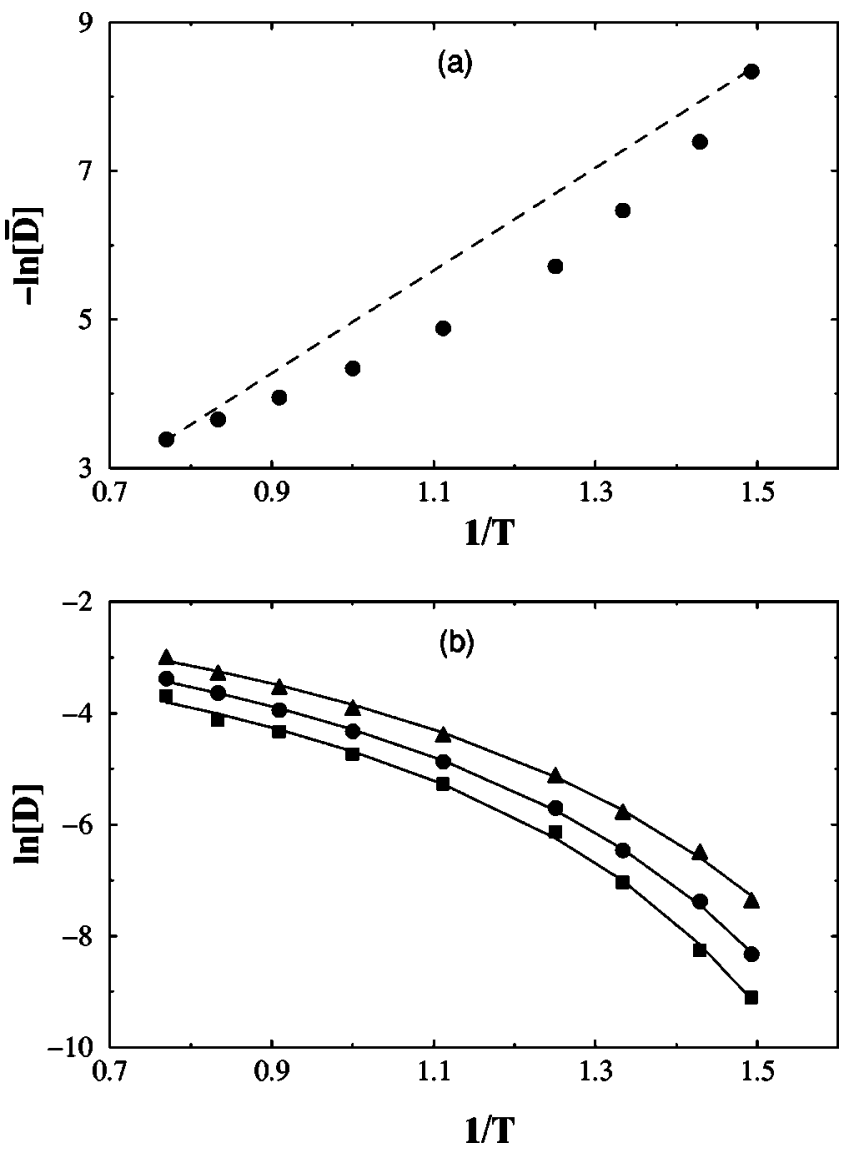

FIG. 3. (a) Temperature dependence of the average diffusion coefficient $(\bar{D}):-\ln [\bar{D}]$ against $1 / T$. The simulated values given by the solid circles show super-Arrhenius behavior. The dashed line gives a guideline to the Arrhenius behavior. (b) Arrhenius plot of diffusion coefficients and the corresponding VFT fits for the smallest $\left(D_{1}\right)$ and largest $\left(D_{10}\right)$ size particles. For comparison, the Arrhenius plot of average diffusion coefficient $(\bar{D})$ with the VFT fit is also shown. Solid triangles and solid squares are the simulation results for the smallest $\left(D_{1}\right)$ and largest $\left(D_{10}\right)$ size particles, respectively. Solid circles are the simulation results for the average diffusion coefficient $(\bar{D})$. The VFT fits in all three cases are represented by the solid lines. The critical temperatures $T_{o}^{D_{1}}, T_{o}^{\bar{D}}$, and $T_{o}^{D_{10}}$ obtained from the VFT fits are $0.46,0.478$, and 0.50 , respectively. Note that the diffusion coefficients are scaled by $\sqrt{\epsilon \bar{\sigma}^{2} / \bar{m}}$.

where the index $i$ stands for the different subsets of particles. $T_{o}^{D_{i}}$ is the critical temperature for the $i$ th species at which the diffusion coefficients $\left(D_{i}\right)$ vanish. The diffusion coefficients for each subsets of particles have been fitted to the above equation and we show the VFT fit to the diffusion coefficients for the smallest $\left(D_{1}\right)$ and largest spheres $\left(D_{10}\right)$ in Fig. 3(b), where $T_{o}^{D_{1}}$ and $T_{o}^{D_{10}}$ are 0.46 and 0.5 . We also show the VFT fit to the average diffusion coefficient $(\bar{D})$ in Fig. 3(b), for comparison, where $T_{o}^{\bar{D}}$ is 0.478 (compared to 0.57 for $T_{o}^{\eta}$ ). Thus the critical temperatures for both the smallest and the largest spheres bracket the critical temperature obtained for the average diffusion coefficient. $T_{o}^{D_{i}}$ depends on the size of the particles and it increases with size of the particles $\bar{R}_{i}$ 


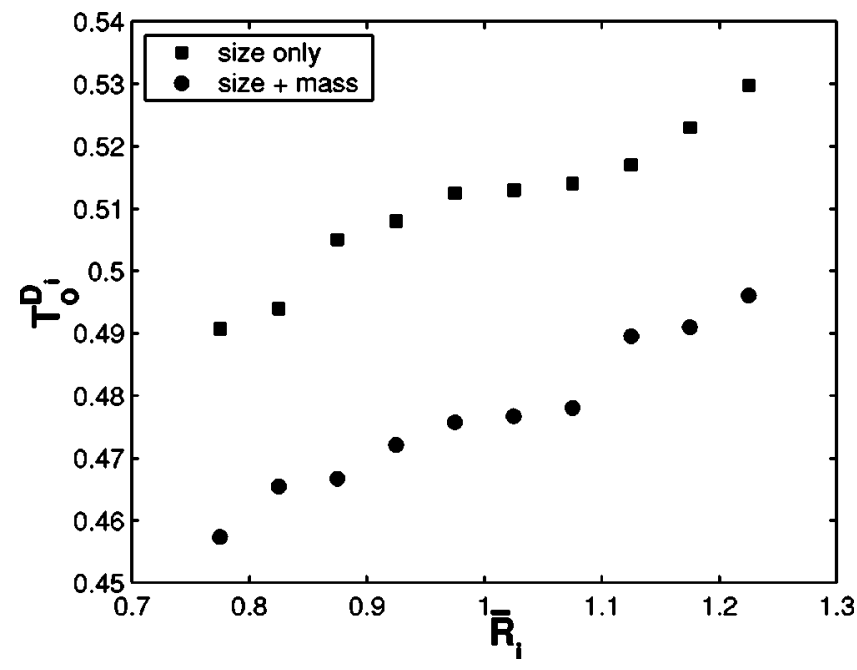

FIG. 4. The critical temperature $\left(T_{o}^{D_{i}}\right)$ obtained from the VFT fit to the different subsets of particles as a function of the mean radius $\left(\bar{R}_{i}\right)$ of the subsets (in units of $\bar{\sigma}$ ) for two different types of polydispersity (polydispersity of both size and mass and polydispersity of size alone). Note that $T_{o}^{D_{i}}$ increases with $\bar{R}_{i}$ in both cases. For details, see the text.

( $\bar{R}_{i}$ is the mean radius of the $i$ th subset). This is shown in Fig. 4. For the largest spheres, the critical temperature $\left(T_{o}^{D_{10}}\right)$ is smaller than the corresponding critical temperature obtained from a VFT fit to the viscosity $\left(T_{o}^{\eta}\right)$. This clearly signifies that near the glass transition the diffusion is partly

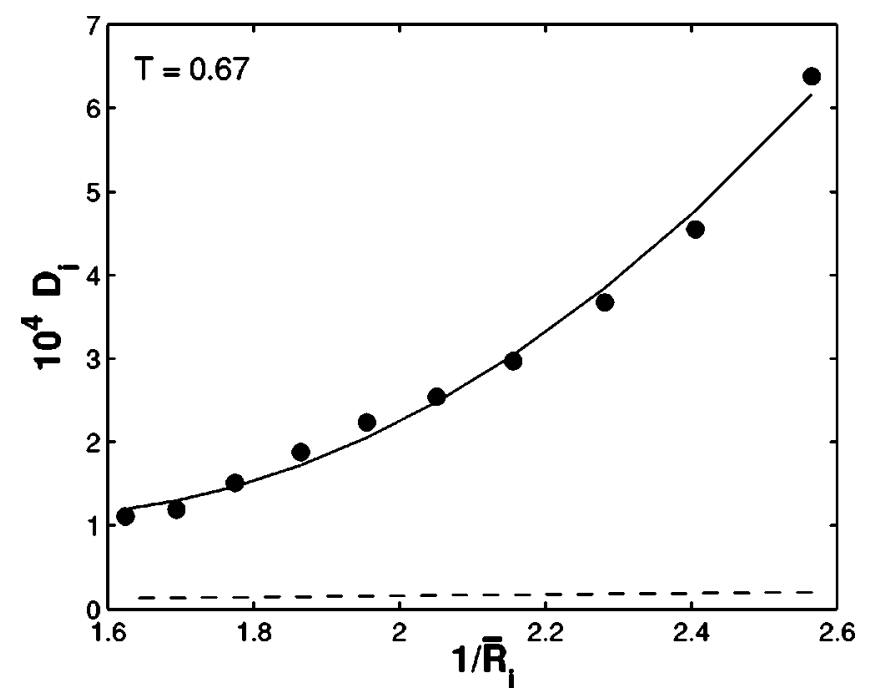

FIG. 5. The diffusion coefficient $\left(D_{i}\right)$ as a function of $1 / \bar{R}_{i}$ at $T=0.67$. The dashed line represents the Stokes-Einstein relation [Eq. (2)] with the stick boundary condition $C=6$. The viscosity $(\eta)$ value is taken from the present simulations. Solid circles are the simulated values and the solid line is the cubic polynomial fit in $1 / \bar{R}_{i}$. The fit parameters are as follows: $D_{i}=0.0011$ $-0.00132\left(1 / \bar{R}_{i}\right)+0.000442\left(1 / \bar{R}_{i}\right)^{2}$. It clearly shows a highly nonlinear size dependence and a marked deviation from Stokesian behavior of the diffusion. Note that the diffusion coefficient $\left(D_{i}\right)$ is scaled by $\sqrt{\epsilon \bar{\sigma}^{2} / \bar{m}}$.
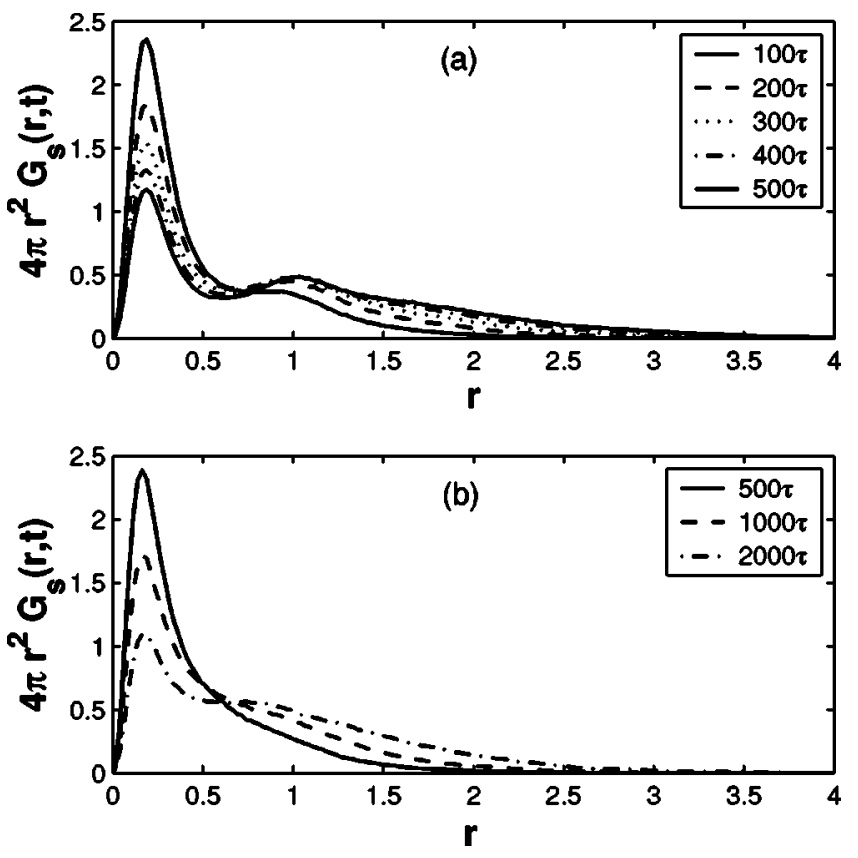

FIG. 6. The van Hove self-correlation function $G_{s}(r, t)$ as a function of the particle displacement $r$ (in units of $\bar{\sigma}$ ) at $T=0.67$ for different values of time $t$ (in units of $\tau=\sqrt{\bar{m} \bar{\sigma}^{2} / \epsilon}=2.2 \mathrm{ps}$ for argon units). (a) For the smallest size particles (subset 1). The occurrence of the second peak at $r \approx 1.0 \bar{\sigma}$ indicates single particle hopping. (b) For the largest size particles (subset 10). Here also a second peak corresponding to single particle hopping develops, but at relatively longer times.

decoupled from the viscosity, and for smaller particles the degree of decoupling is more. The smaller particles remain mobile even when bigger particles are almost frozen.

However, it is interesting to know the influence of purely mass polydispersity, if it is there, as compared to purely size polydispersity, on the observed variation of critical temperature $\left(T_{o}^{D_{i}}\right)$ with size. In order to quantify this, we carried out separate simulations for a system with the same size polydispersity as was considered in our previous case with $s=0.1$, but the mass of all the particles is now considered to be the same and set equal to $\bar{m}$. The critical temperature $\left(T_{o}^{D_{i}}\right)$ obtained from the VFT fit to the diffusion coefficient $\left(D_{i}\right)$ displays similar behavior, as observed in the cases of both size and mass polydispersity (Fig. 4), when plotted as a function of size of the particles $\left(\bar{R}_{i}\right)$-it increases with $\bar{R}_{i}$ (Fig. 4). Compared to the earlier case (of simultaneous size and mass polydispersity), however, the values of the critical temperature for all the particle sizes are higher. These simulations thus suggest that the increase of critical temperature with size is a general observation (i.e., not an effect of mass polydispersity) and may be related to the dynamical heterogeneity induced by geometrical frustration.

It is to be noted that the results that will be presented in the rest of the paper are again for the system of particles with polydispersity of both mass and size. In Fig. 5 we plot the diffusion constants $\left(D_{i}\right)$ against $1 / \bar{R}_{i}$ at the lowest temperature of $T=0.67$ and compared with the well-known hydro- 


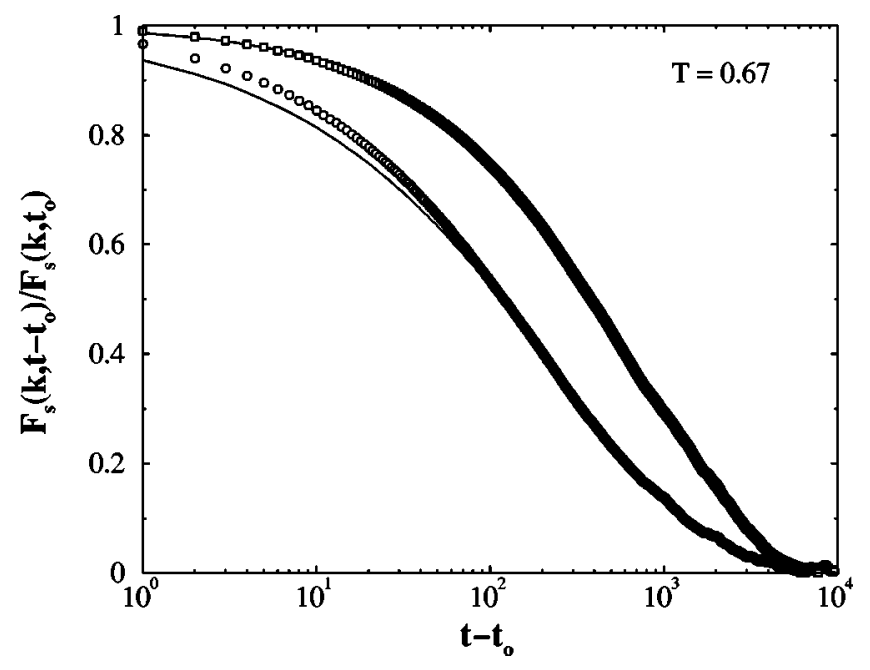

FIG. 7. The self-intermediate scattering function $F_{s}(k, t)$ at $T$ $=0.67$ is shown with a shift in the time origin to $t_{o}=1.0$, and normalized to the value at $t_{o}$, for a fixed value of $k \bar{\sigma} \sim 2 \pi$ for the smallest (subset 1) and largest (subset 10) size particles. This transformation is a convenient way to eliminate the Gaussian dependence at short times [9]. Open circles represent the simulation results for the smallest size particles and open squares represent the simulation results for the largest size particles. The solid lines are the stretched exponential fit [Eq. (6)] to these simulation results. The time constants $\left(\tau_{1}\right.$ and $\left.\tau_{10}\right)$ and the exponents $\left(\beta_{1}\right.$ and $\left.\beta_{10}\right)$ obtained from the fits are $\tau_{1} \simeq 242, \beta_{1} \simeq 0.49, \tau_{10} \simeq 717$, and $\beta_{10}$ $\simeq 0.64$.

dynamic Stokes-Einstein relation [Eq. (2) with $C=6$, the stick boundary condition]. It clearly shows markedly nonStokesian behavior of the diffusion at low temperatures. Interestingly, the fitting to the simulated data points show a highly nonlinear size dependence of the diffusion. This is clear evidence that the breakdown of the SE law is more severe for the smaller size particles. In order to get an estimate of the degree of decoupling (between diffusion and viscosity) for the smallest size particles, we fitted the inverse diffusion coefficient $\left(1 / D_{1}\right)$ versus $\eta / T$. While at high $T$ it asymptotically satisfies the SE relation (the slope is 1 ), the fit to the low temperature data gives the slope $\alpha \approx 0.5$ (that is, diffusion shows the power law behavior $\left.D_{1} \propto \eta^{-0.5}\right)$. It should be noted that the dynamics of a polydisperse liquid is more heterogeneous than that of a monodisperse or bidisperse system due to the different time scales involved for different sizes and masses of the particles [41,44]. The smaller particles are on average faster than others over all time scales. This becomes more prominent at lower temperatures where the relaxation time of the system is very high. At low temperature, the observed nonlinear dependence of diffusion on size may be related to the increase in dynamic heterogeneity in a polydisperse system. While the hydrodynamics cannot explain the nonlinear size dependence even in a dynamically heterogeneous environment, a semiquantitative explanation can be obtained from self-consistent mode coupling theory [52].

A more detailed analysis of the diffusion can be obtained from a closer examination of the self-part of the van Hove
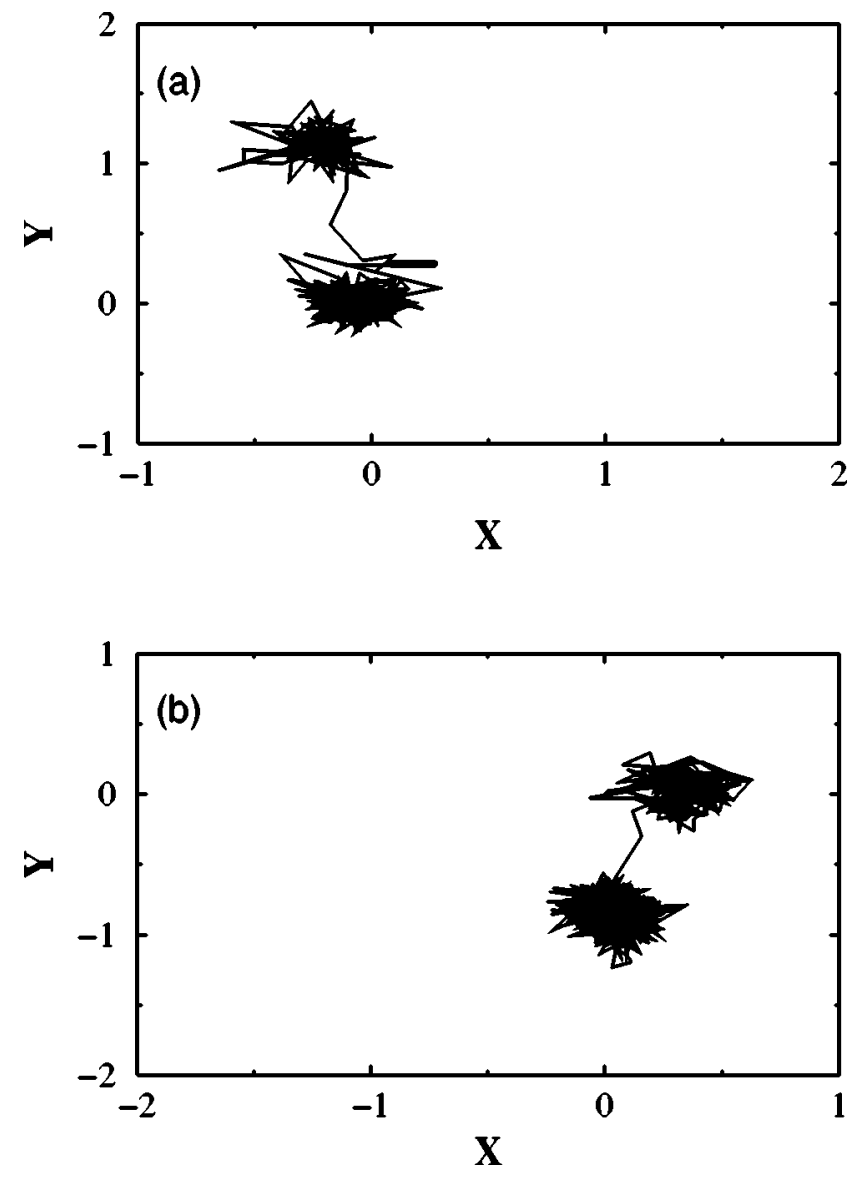

FIG. 8. (a) Projections onto the $x-y$ plane of the trajectory of a typical smallest size particle over a time interval $t=500 \tau$. (b) Projections onto the $x-y$ plane of the trajectory of a typical largest size particle over a time interval $t=2000 \tau$. Note that the time $(t)$ is scaled by $\tau=\sqrt{\bar{m} \bar{\sigma}^{2} / \epsilon}$; it is $2.2 \mathrm{ps}$ if argon units are assumed. For a detailed discussion, see the text.

correlation function $G_{s}(r, t)$. This gives the distribution of the displacements $(r)$ of a particle in a time interval $t$. We calculate $G_{s}(r, t)$ for the smallest $(\sigma=0.75 \bar{\sigma}$ to $0.8 \bar{\sigma})$ and largest $(\sigma=1.2 \bar{\sigma}$ to $1.25 \bar{\sigma})$ particles for different time intervals at $T=0.67$, the lowest temperature investigated. Figures 6(a) and 6(b) show the correlations for the smallest and largest spheres, respectively. For the smallest particles, a gradual development of a well-defined second peak at $r \sim 1.0 \bar{\sigma}$ is clearly visible with increase in time [Fig. 6(a)]. However, for the largest particles, the distribution becomes bimodal at relatively longer time scales [Fig. 6(b)]. The occurrence of the secondary peak, observed also in other model binary mixtures at low temperatures $[18,34,35]$, is evidence of the jump motion in the dynamics of the particles.

To characterize the single particle dynamics further, we evaluated the self-intermediate scattering function $F_{s}(k, t)$, the spatial Fourier transform of $G_{s}(r, t)$, for different subsets of the particles for a fixed value of the reduced wave number $k \bar{\sigma} \sim 2 \pi$ at $T=0.67$. The long time decay of $F_{s}(k, t)$ is well fitted by the Kohlrausch-Williams-Watts (KWW) stretched exponential form: 


$$
F_{s}^{i}(k, t)=\exp \left(-\frac{t}{\tau_{i}}\right)^{\beta_{i}},
$$

where $\tau_{i}$ and $\beta_{i}$ are the relaxation time and the stretching exponent of the $i$ th subset. We find that both $\tau_{i}$ and $\beta_{i}$ increase with an increase in the size of the particles, as was observed earlier by other authors in binary mixtures [14]. The $F_{s}(k, t)$ calculated for the smallest (subset 1) and largest (subset 10) particles along with the KWW fits is shown in Fig. 7. Note that we fit the functions $\left[F_{s}^{i}(k, t\right.$ $\left.\left.-t_{o}\right) / F_{s}^{i}\left(k, t_{o}\right)\right]\left(t>t_{o}\right)$ to the KWW form to quantify their long-time behavior [9]. For the smallest particles, the values of the fitting parameters are found to be $\tau_{1} \simeq 242$ and $\beta_{1}$ $\simeq 0.49$, whereas for the largest particles they are $\tau_{10} \simeq 717$ and $\beta_{10} \simeq 0.64$. The enhanced stretching $\left(\beta_{1}<\beta_{10}\right)$ at long times is due to the greater heterogeneity probed by the smaller size particles than that by the larger size particles during the time scale of decay of their $F_{s}(k, t)$ [53].

In order to determine the extent of the jump-type motion more clearly we follow the trajectory of the individual particles. A close inspection of the simulated trajectory of the smallest and largest particles reveals several interesting features. Figure 8(a) displays the projections onto an $x-y$ plane of the trajectory of a typical smallest size particle over a time interval $\Delta t=500 \tau$, and Fig. 8(b) shows the paths followed by a largest size particle over a time interval $\Delta t=2000 \tau$, both at $T=0.67$. At this temperature the dynamics is dominated by "hopping;" particles remain trapped in transient cages created by the surrounding particles for quite some time and then move significant distances (approximately one interparticle distance) by making a jump to a new cage. For the larger size particles, the jump motion begins to take place at relatively longer time scales [compare also Figs. 6(a) and 6(b)]. Thus, this clearly shows that in a system with particles of all different sizes and masses, the hopping is the dominant diffusive mode for both the smaller and bigger size particles. It is the frequent hopping in the case of smaller size particles that leads to the severe breakdown of the SE relation.

\section{CONCLUSIONS}

In summary, we have presented the results of large scale computer simulations for a supercooled Lennard-Jones poly- disperse system with large variations in temperature at a fixed high pressure. Characteristic of a fragile glass former, a super-Arrhenius temperature dependence is observed for the viscosity and self-diffusion coefficients of different size particles. Interestingly, we find that the critical glass transition temperature (from the VFT relation) for diffusion $\left(T_{o}^{D_{i}}\right)$ increases with the size of the particles and the critical temperature for viscosity $\left(T_{o}^{\eta}\right)$ is larger than that for diffusion. Furthermore, a marked deviation from the Stokesian diffusion is observed where the dependence on the size of the particles is highly nonlinear. At low temperatures, we find that hopping is the dominant mode for mass transport for both the smallest and largest size particles. However, the crossover from continuous Brownian to hopping motion takes place at shorter time scales for the smaller size particles.

In the present system the sizes of all the particles are different. It would be interesting to see whether the jump motion executed by the individual particles occurs over a single energy barrier or takes place via a number of "intermediate" inherent structures in the potential energy landscape. A recent molecular dynamics simulations on a LJ binary mixture [18] showed that such a transition does not correspond to transitions of the system over single energy barriers. In addition, in a deeply supercooled liquid the jump motions are associated with strong nearest-neighbor correlations, in which several neighboring atoms jump at successive close times $[20,21,35]$. It is to be noted that similar correlations have been observed here also. Recently, a computer simulation study of a deeply supercooled binary mixture [22] showed that the local anisotropy in the stress is responsible (at least partly) for the particle hopping. However, the molecular origin of the jump motions observed here (a highly disordered system) is not clear and we are presently pursuing this problem.

\section{ACKNOWLEDGMENTS}

We thank Arnab Mukherjee for many helpful discussions. This work was supported in part by the Council of Scientific and Industrial Research (CSIR), India, and the Department of Atomic Energy (DAE), India. One of the authors (R.K.M) thanks the University Grants Commission (UGC) for financial support.
[1] M. D. Ediger, C. A. Angell, and S. R. Nagel, J. Phys. Chem. 100, 13200 (1996); C. A. Angell, K. L. Ngai, G. B. McKenna, P. F. McMillan, and S. W. Martin, J. Appl. Phys. 88, 3113 (2000); P. G. Debenedetti and F. H. Stillinger, Nature (London) 410, 259 (2001).

[2] M. D. Ediger, Annu. Rev. Phys. Chem. 51, 99 (2000).

[3] E. R. Weeks, J. C. Crocker, A. C. Levitt, A. Schofield, and D. A. Weitz, Science (Washington, DC, U.S.) 287, 627 (2000).

[4] W. K. Kegel and A. van Blaaderen, Science (Washington, DC, U.S.) 287, 290 (2000).

[5] L. A. Deschenes and D. A. Vanden Bout, Science (Washington, DC, U.S.) 292, 255 (2001).
[6] G. Hinze, D. D. Brace, S. D. Gottke, and M. D. Fayer, J. Chem. Phys. 113, 3723 (2000); Phys. Rev. Lett. 84, 2437 (2000).

[7] L.-M. Martinez and C. A. Angell, Nature (London) 410, 663 (2001).

[8] W. Kob and H. C. Andersen, Phys. Rev. Lett. 73, 1376 (1994); Phys. Rev. E 51, 4626 (1995); W. Kob, J. Phys.: Condens. Matter 11, R85 (1999).

[9] S. Sastry, P. G. Debenedetti, and F. H. Stillinger, Nature (London) 393, 554 (1998); S. Sastry, Phys. Rev. Lett. 85, 590 (2000); Nature (London) 409, 164 (2001).

[10] W. Kob, C. Donati, S. J. Plimpton, P. H. Poole, and S. C. 
Glotzer, Phys. Rev. Lett. 79, 2827 (1997); C. Donati, J. F. Douglas, W. Kob, S. J. Plimpton, P. H. Poole, and S. C. Glotzer, ibid. 80, 2338 (1998); C. Donati, S. C. Glotzer, P. H. Poole, W. Kob, and S. J. Plimpton, Phys. Rev. E 60, 3107 (1999); S. C. Glotzer and C. Donati, J. Phys.: Condens. Matter 11, A285 (1999).

[11] D. Thirumalai and R. D. Mountain, J. Phys. C 20, L399 (1987); R. D. Mountain and D. Thirumalai, Phys. Rev. A 36, 3300 (1987); A. I. Mel'cuk, R. A. Ramos, H. Gould, W. Klein, and R. D. Mountain, Phys. Rev. Lett. 75, 2522 (1995).

[12] A. Heuer, Phys. Rev. Lett. 78, 4051 (1997); S. Buchner and A. Heuer, Phys. Rev. E 60, 6507 (1999); J. Qian, R. Hentschke, and A. Heuer, J. Chem. Phys. 110, 4514 (1999); 111, 10177 (1999).

[13] C. Oligschleger and H. R. Schober, Phys. Rev. B 59, 811 (1999); D. Caprion, J. Matsui, and H. R. Schober, Phys. Rev. Lett. 85, 4293 (2000).

[14] D. N. Perera and P. Harrowell, Phys. Rev. E 59, 5721 (1999); J. Chem. Phys. 111, 5441 (1999).

[15] L. Angelani, G. Parisi, G. Ruocco, and G. Viliani, Phys. Rev. Lett. 81, 4648 (1998).

[16] A. Mukherjee, S. Bhattacharyya, and B. Bagchi, J. Chem. Phys. 116, 4577 (2002).

[17] U. Bengtzelius, W. Gotze, and A. Sjolander, J. Phys. C 17, 5915 (1984); W. Gotze and L. Sjogren, Rep. Prog. Phys. 55, 241 (1992).

[18] T. B. Schroder, S. Sastry, J. C. Dyre, and S. C. Glotzer, J. Chem. Phys. 112, 9834 (2000).

[19] L. Angelani, R. Di Leonardo, G. Ruocco, A. Scala, and F. Sciortino, Phys. Rev. Lett. 85, 5356 (2000).

[20] H. Miyagawa, Y. Hiwatari, B. Bernu, and J. P. Hansen, J. Chem. Phys. 88, 3879 (1988).

[21] S. Bhattacharyya, A. Mukherjee, and B. Bagchi, J. Chem. Phys. 117, 2741 (2002).

[22] S. Bhattacharyya and B. Bagchi, Phys. Rev. Lett. 89, 025504 (2002).

[23] J. A. Hodgdon and F. H. Stillinger, Phys. Rev. E 48, 207 (1993).

[24] G. Tarjus and D. Kivelson, J. Chem. Phys. 103, 3071 (1995).

[25] C. Z.-W. Liu and I. Oppenheim, Phys. Rev. E 53, 799 (1996).

[26] E. Rossler, Phys. Rev. Lett. 65, 1595 (1990).

[27] M. T. Cicerone, F. R. Blackburn, and M. D. Ediger, J. Chem. Phys. 102, 471 (1995); M. T. Cicerone and M. D. Ediger, ibid. 104, 7210 (1996).

[28] F. Fujara, B. Geil, H. Sillescu, and G. Fleischer, Z. Phys. B: Condens. Matter 88, 195 (1992); I. Chang et al., J. Non-Cryst. Solids 172-174, 248 (1994).

[29] D. Thirumalai and R. D. Mountain, Phys. Rev. E 47, 479 (1993)
[30] R. Yamamoto and A. Onuki, Phys. Rev. E 58, 3515 (1998).

[31] R. Yamamoto and A. Onuki, Phys. Rev. Lett. 81, 4915 (1998).

[32] D. N. Perera and P. Harrowell, Phys. Rev. Lett. 81, 120 (1998).

[33] C. D. Michele and D. Leporini, Phys. Rev. E 63, 036701 (2001).

[34] J. N. Roux, J. L. Barrat, and J. P. Hansen, J. Phys.: Condens. Matter 1, 7171 (1989); J. L. Barrat, J. N. Roux, and J. P. Hansen, Chem. Phys. 149, 197 (1990).

[35] G. Wahnstrom, Phys. Rev. A 44, 3752 (1991).

[36] W. Kob and J. L. Barrat, Phys. Rev. Lett. 78, 4581 (1997); K. Vollmayr-Lee, W. Kob, K. Binder, and A. Zippelius, J. Chem. Phys. 116, 5158 (2002).

[37] P. N. Pusey, Liquids, Freezing and Glass Transitions, Proceedings of the Les Houches Summer School of Theoretical Physics, Session LI, 1989, edited by J. P. Hansen, D. Levesque, and J. Zinn-Justin (North-Holland, Amsterdam, 1991); P. N. Pusey and W. van Megen, Phys. Rev. Lett. 59, 2083 (1987); P. N. Segre, S. P. Meeker, P. N. Pusey, and W. C. K. Poon, ibid. 75, 958 (1995).

[38] A. P. Gast and W. B. Russel, Phys. Today 51, 24 (1998).

[39] D. A. Kofke and P. G. Bolhuis, Phys. Rev. E 59, 618 (1999).

[40] D. J. Lacks and J. R. Wienhoff, J. Chem. Phys. 111, 398 (1999).

[41] R. P. Sear, J. Chem. Phys. 113, 4732 (2000).

[42] B. Doliwa and A. Heuer, Phys. Rev. Lett. 80, 4915 (1998).

[43] K. N. Pham et al., Science (Washington, DC, U.S.) 296, 104 (2002).

[44] N. Kiriushcheva and P. H. Poole, Phys. Rev. E 65, 011402 (2001).

[45] M. P. Allen and D. J. Tildesley, Computer Simulation of Liquids (Oxford University Press, Oxford, 1987).

[46] G. J. Martyna, D. J. Tobias, and M. L. Klein, J. Chem. Phys. 101, 4177 (1994); H. C. Andersen, ibid. 72, 2384 (1980); S. Nosé, Mol. Phys. 52, 255 (1984); W. G. Hoover, Phys. Rev. A 31, 1695 (1985).

[47] G. J. Martyna, M. E. Tuckerman, and M. L. Klein, J. Chem. Phys. 97, 2635 (1992).

[48] M. E. Tuckerman, G. J. Martyna, and B. J. Berne, J. Chem. Phys. 97, 1990 (1992).

[49] G. J. Martyna, M. E. Tuckerman, D. J. Tobias, and M. L. Klein, Mol. Phys. 87, 1117 (1996).

[50] J. P. Hansen and I. R. McDonald, Theory of Simple Liquids (Academic, London, 1986).

[51] C. A. Angell, Science (Washington, DC, U.S.) 267, 1924 (1995).

[52] B. Bagchi and S. Bhattacharyya, Adv. Chem. Phys. 116, 67 (2001).

[53] R. K. Murarka, S. Bhattacharyya, and B. Bagchi, J. Chem. Phys. 117, 10730 (2002). 